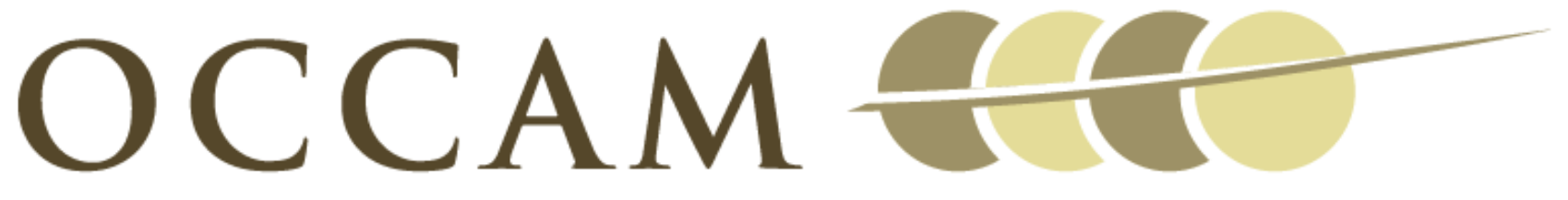

OXFORD CENTRE FOR COLLABORATIVE APPLIED MATHEMATICS

Report Number 10/10

On the mechanical stability of growing arteries

by

Alain Goriely and Rebecca Vandiver

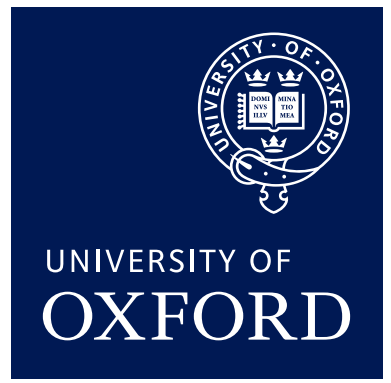

Oxford Centre for Collaborative Applied Mathematics Mathematical Institute

24 - 29 St Giles'

Oxford

OX1 3LB

England 



\title{
On the mechanical stability of growing arteries
}

\author{
Alain Goriely* and Rebecca Vandiver \\ Program in Applied Mathematics and ${ }^{*}$ Department of Mathematics, \\ University of Arizona, Building \#89, Tucson, AZ 85721 \\ E-mail: goriely@ math.arizona.edu \\ Corresponding author: A. G.
}

March 8, 2010

- Abstract Arteries are modeled, within the framework of nonlinear elasticity, as incompressible two-layer cylindrical structures that are residually stressed through differential growth. These structures are loaded by an axial force, internal pressure and have nonlinear, anisotropic, hyperelastic response to stresses. Parameters for this model are directly related to experimental observations. The possible role of axial residual stress in regulating stress in arteries and preventing buckling instabilities is investigated. It is shown that axial residual stress lowers the critical internal pressure leading to buckling and that a reduction of axial loading may lead to a buckling instability which may eventually lead to arterial tortuosity.

\section{Introduction}

Arteries are the blood vessels that carry blood from the heart to organs and body tissues. The arterial system is a highly complicated and regulated mechanical system sustaining varying pressures, loads, and stresses. Starting in the 1960's many different puzzling experimental observations revealed that large arteries have mechanical responses that cannot be readily understood by elementary mechanics. Patel and Fry showed that during the cardiac cycle, and despite large variations of pressure, the end-to-end distance of major arteries of dogs remains mostly unchanged [1, 2]. Moreover, during regular homeostatic functions, arteries are tethered to other tissues and they operate under a large stretch of about 1.4 to 1.6 [3]. They will therefore naturally retract to their preferred rest length when cut. However, even in their rest length and in the absence of pressure, Bergel noted that arteries are still stressed (see [4]). The importance and existence of these residual stresses (stresses that persist in the absence of external or body loads) in arteries were revealed by the work of Fung and Vaishnav and Vossoughi in the 1980's [5, 6, 7, 8]. Residual stresses in arteries are demonstrated by first slicing a disk out of an artery and by observing how this disk opens when a radial cut is made. The opening that results from the cut is measured by the so-called "opening angle", a kinematic measure of residual strains [9]. The stresses associated with the opening angle are known to play a fundamental role in regulating transmural stress gradient and lowering circumferential stress at the inner walls $[10,11]$. Most of the models formulated to study residual stress have considered the two-dimensional field associated with the opening angle without accounting for the effect of the axial direction ([12], [11], [13]). Experimental observations ([14], [15]) show that arteries have axial residual stress. These studies have qualitatively suggested the importance of residual stress in the axial direction, but the effect of axial residual stress and the axial pre-stretch still remains to be elucidated.

Residual stresses in arteries are believed to be the result of differential growth and remodeling. It has been known since the early work of R. Thoma in 1893 (see [16]) that the magnitude of blood flow in chick embryo blood vessels regulates the vessels' diameters and that this effect is mediated through shear stress on the inner arterial wall. Similarly, the magnitude of the pressure regulates the vessels' thicknesses. The mechanical regulation of artery thickness and diameter has been verified experimentally [17, 18]. Arteries also respond to changes in axial loading; a sustained increase in axial loading tends to lengthen arteries in culture [19] and in vivo [20] suggesting that axial stress (or strain) is also regulated towards a homeostatic value $[21]$. 
The effect of axial unloading is quite different. Recently, Jackson et al. [22] showed that partial unloading of axial tension can induce arterial tortuosity. More explicitly, they studied the relationship between the development of tortuosity and mechanical forces imposed on arterial tissue. The axial strain in rabbit carotid arteries was reduced from $62 \pm 2 \%$ and maintained at $33 \pm 2 \%$ for twelve weeks. After this time period, all vessels became tortuous due to tissue growth and remodeling, and they concluded that a substantial axial strain is necessary to sustain arterial stability. Arterial tortuosity is a well-known condition that can occur in response to abnormal growth stimuli or genetic defects $[23,24,25,26,27,28]$. The stability of arteries under this load is essential to maintaining normal arterial function. Instability can lead to serious complications including stroke, vertigo, blackout, fainting, and persistent tinnitus [29, 30, 31], yet the biomechanical basis of why arterial buckling occurs is not well understood. Further experimental studies on the instability of arteries were conducted by Han [32] who set porcine carotid arteries at a fixed axial stretch and gradually pressurized the arteries until significant mechanical buckling occurred. The results supported by a linear stability analysis [33] showed that the critical pressure leading to buckling is directly related to the axial stretch ratio, very close to a linear relationship in the tested range.

The purpose of this article is to study theoretically the mechanics and stability of arteries including radial and axial residual stresses and investigate the possibility of mechanical buckling in large deformations. Accordingly, in Section 2, we use a mechanical two-layer model of anisotropic hyperelastic arteries proposed by Holzapfel et al. [13] together with a description of residual stress through a multiplicative decomposition of the growth tensor as proposed by Taber [34]. Then, in Section 3, we study the response of arteries in large deformation and in Section 4, we perform a stability analysis by using the method proposed by Ben Amar and Goriely [35]. We use these tools to answer the following questions

1. What are the mechanical factors (anisotropy, residual stress, ...) responsible for the constancy of artery length in normal physiological regime?

2. Could axial off-loading of an artery result in mechanical buckling?

3. What is the role of residual stresses in arterial stability?

We show that the constancy in artery length is due to the exponential nonlinearity as modeled by the Fung material. The stability analysis reveals that both axial off-loading and an increase in the internal pressure can lead to mechanical buckling and lastly, we observe that axial residual stress plays an important role in preventing buckling.

\section{A mechanical model of growing arteries}

\subsection{General Kinematics}

We consider a continuous body whose reference configuration is defined by $\mathcal{B}_{0}$ (See Fig.1) with position vector $\mathbf{X}$. The body is deformed to the current configuration, $\mathcal{B}_{f}$ where the position of a material point $\mathbf{X}$ is $\mathbf{x}=\boldsymbol{\chi}(\mathbf{X}, t)$. The deformation gradient, $\mathbf{F}(\mathbf{X}, t)=\operatorname{Grad} \boldsymbol{\chi}$ (taken in the reference configuration), relates a material segment in the reference configuration to the same segment in the current configuration. Here we use the fundamental assumption of morpho-elasticity as first stated by Rodriguez, Hoger, and McCulloch [36]. That is, we assume that the current state of an artery (or a grown body in general) is the result of a local growth step characterized by a growth tensor $\mathbf{G}$ followed by an elastic step characterized by an elastic tensor $\mathbf{A}$ so that the product of these two tensors gives the deformation gradient. That is, we have

$$
\mathbf{F}(\mathbf{X}, t)=\mathbf{A}(\mathbf{X}, t) \cdot \mathbf{G}(\mathbf{X}, t) .
$$

While $\mathbf{F}$ is the gradient of a deformation, this is not necessarily true for $\mathbf{A}$ and $\mathbf{G}$. The growth tensor, $\mathbf{G}$ describes locally the generation (or removal) of new material points and the elastic deformation tensor, A, describes the pure deformation resulting from stresses. As shown in Figure 1, the growth tensor $\mathbf{G}(\mathbf{X}, t)$ maps $\mathcal{B}_{0}$ to the virtual configuration $\mathcal{V}$ which is locally stress-free. In order to maintain continuity of the body, the elastic deformation is introduced which in turn causes residual stress in the grown body $\mathcal{B}_{f}$ (the extra configuration $\mathcal{B}_{1}$ will be discussed shortly). 


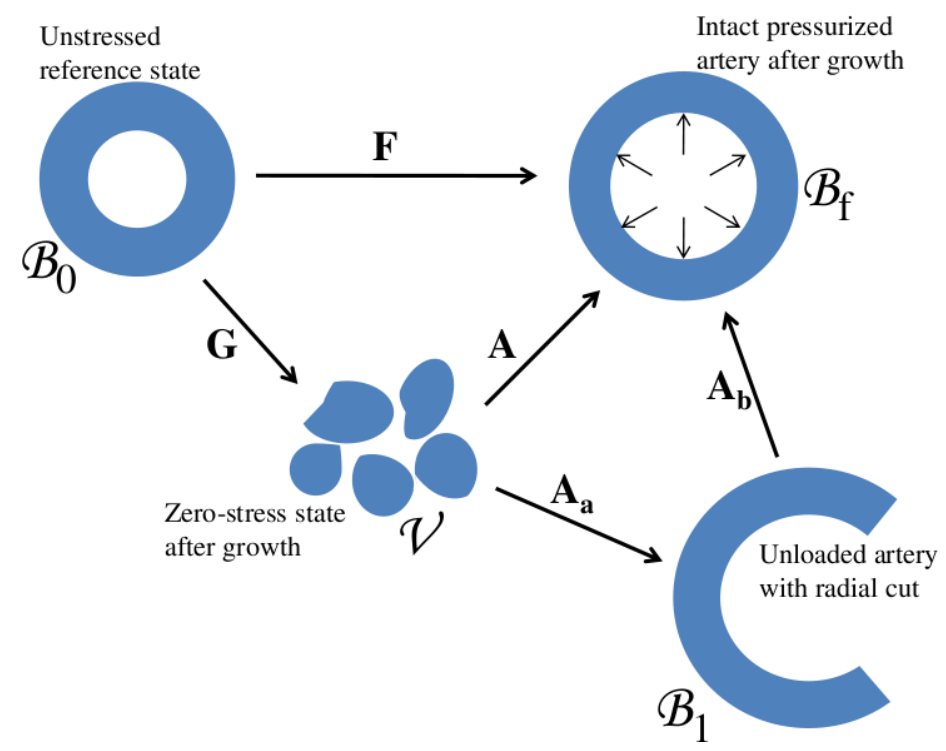

Figure 1: Decomposition into a single opened ring due to a radial cut.

\subsection{Cylindrical deformations}

For the particular case of an artery, we consider the idealized cylindrical geometry and a two-layered cylinder (see discussion in Section 2.4) with an inner cylinder of initial radii $A$ and $B$ and an outer layer of initial radii $B$ and $C$ (shown in Figure 2). Before bifurcation, we consider a finite deformation in which the cylinder is allowed to grow and deform while remaining cylindrical. This is a semi-inverse problem for which all relevant mechanical and geometrical quantities can be computed. After bifurcation, we will consider incremental solutions around the finite cylindrical deformation that do not respect the cylindrical symmetry. The finite deformation of each cylindrical shell is then

$$
\mathbf{x}_{i, o}=\chi_{i, o}\left(\mathbf{X}_{i, o}, t\right)
$$

where

$$
\mathbf{X}_{i, o}=\left(R_{i, o}, \Theta_{i, o}, Z_{i, o}\right), \quad \mathbf{x}_{i, o}=\left(r_{i, o}, \theta_{i, o}, z_{i, o}\right),
$$

and $r_{i, o}=r_{i, o}\left(R_{i, o}\right), \Theta_{i, o}=\theta_{i, o}$, and $Z_{i, o}=\lambda z_{i, o}$. The subscripts $i$ and $o$ refer to the inner and outer layers, respectively. 


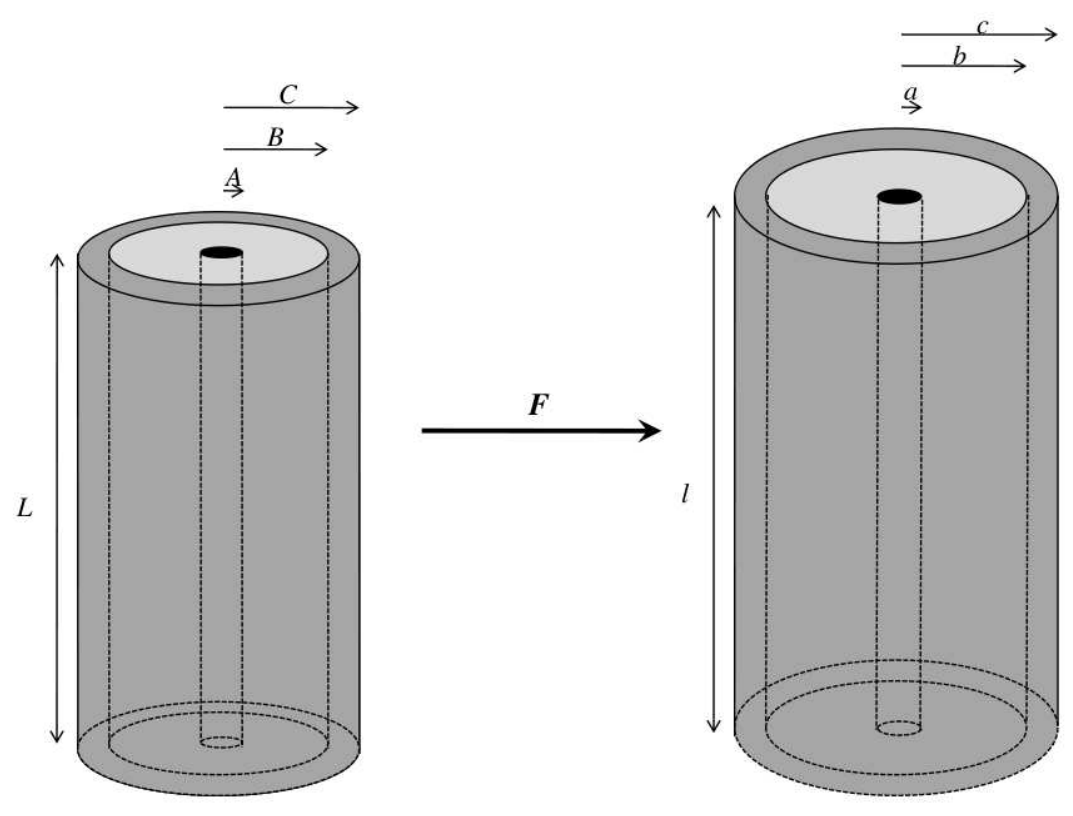

Figure 2: Geometry in the initial and grown configurations.

The geometric deformation in the usual cylindrical coordinates (radius $r$, angle $\theta$, height $z$ ) is

$$
\begin{aligned}
\mathbf{F}_{i, o} & =\operatorname{Grad}\left(\boldsymbol{\chi}_{i, o}\right) \\
& =\operatorname{diag}\left(r_{i, o}^{\prime}, r_{i, o} / R_{i, o}, \lambda\right),
\end{aligned}
$$

where the prime denotes differentiation with respect to $R_{i, o}$, and $\lambda$ is the axial stretch of the cylinder such that $l=\lambda L$. Similarly, the growth tensor is given by

$$
\mathbf{G}_{i, o}=\operatorname{diag}\left(\gamma_{r_{i, o}}, \gamma_{\theta_{i, o}}, \gamma_{z_{i, o}}\right) .
$$

The values of the parameters in the growth tensor will be determined from known values of the residual strain (through the opening angle) in Section 3.1.

\subsection{Mechanical Equilibrium}

We now consider the balance of forces acting on $\mathcal{B}_{f}$. Since we consider the body in the current configuration, the balance of forces follows directly from Cauchy's equation for the Cauchy stress tensor $\mathbf{T}$, that is

$$
\operatorname{div}\left(\mathbf{T}_{i, o}^{\mathrm{T}}\right)=0, \quad \mathbf{T}_{i, o}^{\mathrm{T}}=\mathbf{T}_{i, o},
$$

where the divergence is taken with respect to $\mathbf{x}$ in the current configuration and the body forces are assumed to be negligible. The solutions to the equilibrium equations must also satisfy the conditions imposed on the boundary.

We assume that the body is hyperelastic. That is, the material can be described by a strain energy function $W_{i, o}=W_{i, o}\left(\mathbf{A}_{i, o}\right)$ and the Cauchy stress is related to the elastic deformation by

$$
\mathbf{T}_{i, o}=J^{-1} \mathbf{A}_{i, o} \cdot \frac{\partial W_{i, o}}{\partial \mathbf{A}_{i, o}}-p_{i, o} \mathbf{1},
$$

where $p_{i, o}$ is a Lagrange multiplier associated with the internal constraint of incompressibility and $J=\operatorname{det}(\mathbf{A})$ represents the change of volume due to the elastic deformation. For an incompressible material, $J=1$ and $p$ is a Lagrange multiplier. If the material is compressible, then $p=0$. 


\subsection{Elastic response}

The arterial wall consists of three layers, each containing specific histological features: the intima (the innermost layer), the media, and the adventitia (the outer layer). The intima is known to have negligible (solid) mechanical contributions [37] and therefore the arterial wall is approximated as a two-layer structure containing the media and adventitia.

Numerous constitutive models have been proposed for arteries (see review in [38]). Most models [39, $40,41]$ are formulated by fitting the constitutive equations to experimental data which is referred to as a phenomenological approach. Here, we start with the multi-layer model for arteries introduced by Holzapfel et al. [13]. This model has the advantage of being structural in the sense that it incorporates layer-specific histological features. Each layer is reinforced with collagen fibers with preferred directions that render the material anisotropic [42]. The model accounts for the orientations of the collagen fibers in each layer. The contribution of collagen fibers in the arterial wall is considered to be negligible at low pressures and therefore, the mechanical response is assumed to be isotropic. However, at higher pressures, the collagen fibers are stretched and the resulting mechanical response is anisotropic. For each layer Holzapfel et al. [13] suggest a strain-energy of the form

$$
W\left(I_{1}, I_{4}, I_{6}\right)=W_{\text {iso }}\left(I_{1}\right)+W_{\text {aniso }}\left(I_{4}, I_{6}\right),
$$

where the invariants $I_{1}, I_{4}, I_{6}$ of the right Cauchy-Green tensor $\mathbf{C}=\mathbf{F}^{\mathrm{T}} \cdot \mathbf{F}$ are given by

$$
I_{1}=\operatorname{tr} \mathbf{C}, \quad I_{4}=\mathbf{C}: \mathbf{M} \otimes \mathbf{M}, \quad I_{6}=\mathbf{C}: \mathbf{M}^{\prime} \otimes \mathbf{M}^{\prime} .
$$

The invariants $I_{4}$ and $I_{6}$ are the squares of the stretches in the respective directions of the two families of collagen fibers. The unit vectors $\mathbf{M}$ and $\mathbf{M}$ ' describe the orientations of these fibers and these vectors are defined for each layer in the reference configuration. In the usual cylindrical coordinate system, the components of the direction vectors are

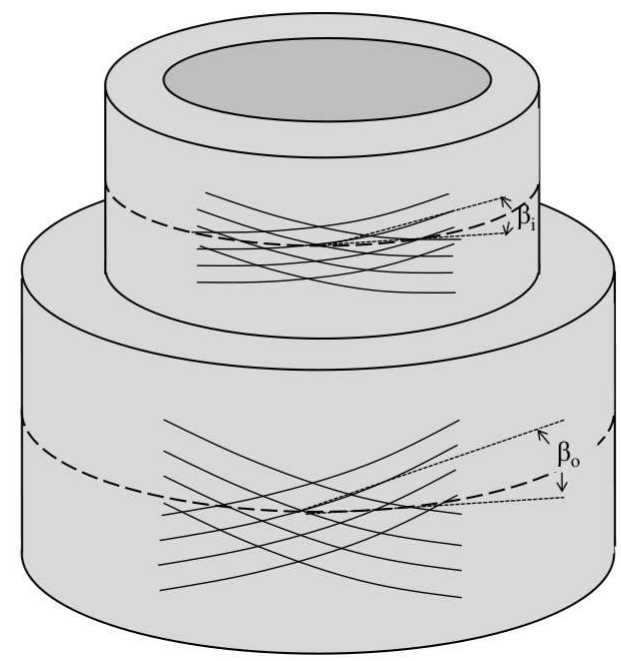

Figure 3: Schematic of the angle defined between the collagen fibers and the circumferential direction.

$$
\left[\begin{array}{c}
M_{r}^{(j)} \\
M_{\theta}^{(j)} \\
M_{z}^{(j)}
\end{array}\right]=\left[\begin{array}{c}
0 \\
\cos \beta_{j} \\
\sin \beta_{j}
\end{array}\right],\left[\begin{array}{c}
M_{r}^{\prime(j)} \\
M_{\theta}^{\prime(j)} \\
M_{z}^{\prime(j)}
\end{array}\right]=\left[\begin{array}{c}
0 \\
\cos \beta_{j} \\
-\sin \beta_{j}
\end{array}\right], \quad j=i, o,
$$

where the subscripts $i$ and $o$ refer to the inner layer (the media) and the outer layer (the adventitia), respectively, and the fibers are assumed to lie in the tangent plane. The angle that occurs between the collagen fibers and the circumferential direction is denoted by $\beta_{j}$ (see Figure 3). The isotropic and anisotropic responses in (9) are given by

$$
W_{i s o}=\frac{\mu}{2}\left(I_{1}-3\right)
$$




$$
W_{\text {aniso }}\left(I_{4}, I_{6}\right)=\frac{k_{1}}{2 \nu} \sum_{n=4,6}\left\{e^{\nu\left(I_{n}-1\right)^{2}}-1\right\}
$$

where the material parameter $k_{1}$ has the dimension of stress and $\nu$ is a dimensionless parameter. The anisotropic term contributes to the mechanical response only when the fibers are extended, in other words when either $I_{4}>1$ or $I_{6}>1$ or both. Typical material and geometrical data used here are from a rabbit carotid artery [43] $\left(A=0.71, B=0.97, C=1.10, \mu_{i}=3.0000 \mathrm{kPa}, k_{1 i}=2.3632 \mathrm{kPa}, \nu_{i}=0.8393, \mu_{o}=\right.$ $\left.0.3000 \mathrm{kPa}, k_{1 o}=0.5620 \mathrm{kPa}, \nu_{o}=0.7112, \beta_{i}=29.0^{\circ}, \beta_{o}=62.0^{\circ}\right)$. The cylinder is subjected to an internal pressure, $P$, and therefore the axial equilibrium and the boundary conditions at the inner and outer surfaces yield

$$
\begin{gathered}
2 \int_{a}^{c} r t_{z} d r=P a^{2}, \\
t_{r}(a)=-P, \quad t_{r}(c)=0,
\end{gathered}
$$

where $t_{r}$ and $t_{z}$ are the Cauchy stresses in the radial and axial directions, respectively. Note that the cylindrical symmetry will be preserved in the finite deformation because the two families of fibers are mechanically equivalent and are arranged symmetrically.

\section{Finite deformation}

The strain-energy function $W_{i, o}$ relates the elastic deformation to the Cauchy stress tensor $\mathbf{T}=\operatorname{diag}\left(t_{r}, t_{\theta}, t_{z}\right)$ by

$$
\mathbf{T}_{i, o}=\mathbf{A}_{i, o} \cdot \frac{\partial W_{i, o}}{\mathbf{A}_{i, o}}-p_{i, o} \mathbf{1}
$$

which can be used in the mechanical equilibrium equation $\operatorname{div}\left(\mathbf{T}_{i, o}\right)=0$ to obtain

$$
\frac{\partial t_{r}(r)}{\partial r}= \begin{cases}\frac{\alpha_{i} \partial_{\alpha_{i}} \hat{W}_{i}}{r} & a \leq r \leq b, \\ \frac{\alpha_{o} \partial_{\alpha_{o}} \hat{W}_{o}}{r} & b \leq r \leq c,\end{cases}
$$

where $\hat{W}_{i, o}\left(\alpha_{i, o}\right)=W_{i, o}\left(\gamma_{z_{i, o}} /\left(\alpha_{i, o} \lambda\right), \alpha_{i, o}, \lambda / \gamma_{z_{i, o}}\right)$. Assuming the boundary conditions of zero normal traction on the outer boundary and an internal pressure, $P$, at the inner boundary $\left(t_{r}(a)=-P\right.$ and $\left.t_{r}(c)=0\right)$, we integrate Equation (17) to obtain an equation for the radial stress

$$
t_{r}(r)= \begin{cases}-P+\int_{a}^{r} \frac{\alpha_{i} \partial_{\alpha_{i}} \hat{W}_{i}}{r} & a \leq r \leq b, \\ -\int_{r}^{c} \frac{\alpha_{o} \partial_{\alpha_{o}} \hat{W}_{o}}{r} & b \leq r \leq c .\end{cases}
$$

The axial stress is given by the $z$ component of Equation (16), that is,

$$
t_{z}(r)=t_{r}+\frac{\lambda}{\gamma_{z_{i, o}}} W_{3}-\frac{\gamma_{z_{i, o}}}{\lambda \alpha}
$$

where $W_{1}$ and $W_{3}$ are the derivatives of $W$ with respect to its first and third variables. At the surface of stress discontinuity at $r=b$, the associated tractions need to be equal but opposite, which requires the radial stress to be continuous across $r$, that is

$$
t_{r}\left(b^{+}\right)=t_{r}\left(b^{-}\right) .
$$

The two free parameters $a$ and $\lambda$ are obtained by solving simultaneously the boundary condition (20) for the given resultant axial load $N$,

$$
N=2 \pi \int_{a}^{c} r t_{z}(r) d r=\pi a^{2} P,
$$

and the solution for the configuration $\mathcal{B}_{f}$ is completely determined. The reduced axial force, $F$, is given by $F=N-\pi a^{2} P=0$, that is there is no net force acting on the cylinder. 


\subsection{Residual stresses}

We use the multiplicative decomposition of morpho-elasticity to characterize the residual stress present in an artery. To do so, we use the method of Taber [44] to determine the form of the growth tensor compatible with a given residual stress or residual strain fields. In our case, we use data on the opening angle from a radial cut on a thin ring of artery to determine the value of the growth tensor. The basic idea [36] is to assume that the current state of an unloaded artery is the result of a local growth step characterized by a growth tensor $\mathbf{G}$ followed by an elastic step characterized by an elastic tensor $\mathbf{A}$ so that the product of these two tensors gives the deformation gradient. That is, we assume the decomposition $\mathbf{F}=\mathbf{A} \cdot \mathbf{G}$. The material elements in the configuration $\mathcal{V}$ are reassembled into the configuration $\mathcal{B}_{1}$, which represents a single opened ring due to a radial cut (Figure 1). The deformation $\mathbf{A}_{a}$ to configuration $\mathcal{B}_{1}$ will contain residual stress if the elements of $\mathcal{V}$ are geometrically incompatible. Lastly, the deformation $\mathbf{A}_{b}$ bends the ring closed and the ring is subjected to an internal pressure $P$ to produce the final configuration $\mathcal{B}_{f}$.

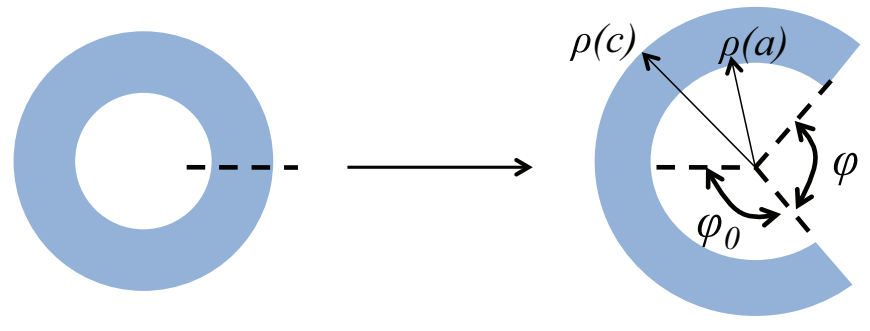

Figure 4: Schematic of a radial cut in an artery and the corresponding opening angle $\varphi$.

The cylindrical polar coordinates in $\mathcal{B}_{1}$ are given by $(\rho, \vartheta, \zeta)$. The elastic tensor $\mathbf{A}$ can be decomposed into the product of two tensors, $\mathbf{A}=\mathbf{A}_{a} \cdot \mathbf{A}_{b}$, where

$$
\mathbf{A}_{a}=\operatorname{diag}\left(\alpha_{a \rho}, \alpha_{a \vartheta}, \alpha_{a \zeta}\right)
$$

and

$$
\mathbf{A}_{b}=\operatorname{diag}\left(\alpha_{b \rho}, \alpha_{b \vartheta}, \alpha_{b \zeta}\right) .
$$

The solution for configuration $\mathcal{B}_{f}$ is calculated as described in Section 3. Once this solution is known, the geometry and corresponding stresses in the cut ring can be determined as follows. The total deformation in terms of the material coordinates in $\mathcal{B}_{1}$ is given by

$$
r=r(\rho), \quad \theta=\pi \vartheta / \varphi_{0}, \quad z=\Lambda \zeta,
$$

where $\Lambda$ is the axial stretch and $\varphi_{0}$ is the angle related to the opening angle $\varphi=2\left(\pi-\varphi_{0}\right)$ defined in Figure 4. The kinematic relationships are given by

$$
\begin{gathered}
\alpha_{a \rho}=\lambda_{r}\left(\alpha_{b \rho} \gamma_{r}\right)^{-1}, \quad \alpha_{a \vartheta}=\lambda_{\theta}\left(\alpha_{b \vartheta} \gamma_{\theta}\right)^{-1}, \quad \alpha_{a z}=\lambda_{z}\left(\alpha_{b \zeta} \gamma_{z}\right)^{-1}, \\
\alpha_{b \rho}=\partial r / \partial \rho, \quad \alpha_{b \vartheta}=\pi r / \varphi_{0} \rho, \quad \alpha_{b \zeta}=\Lambda,
\end{gathered}
$$

and the incompressibility conditions are given by

$$
\alpha_{a \rho} \alpha_{a \vartheta} \alpha_{a z}=\alpha_{b \rho} \alpha_{b \vartheta} \alpha_{b z}=1 .
$$

The equilibrium equation is

$$
\frac{\partial t_{\rho}}{\partial \rho}+\frac{t_{\rho}-t_{\vartheta}}{\rho}=0
$$

where the Cauchy stress components $t_{i}$ in $\mathcal{B}_{1}$ are given by

$$
t_{i}=\alpha_{a i} \frac{\partial W}{\partial \alpha_{a i}}-\hat{p}
$$


The subscript $i$ corresponds to the parameters $\rho, \vartheta$, and $\zeta, \hat{p}$ is the Lagrange multiplier in $\mathcal{B}_{1}$, and the strainenergy density function is $W=W\left(\alpha_{a \rho}, \alpha_{a \vartheta}, \alpha_{\zeta}\right)$. In the unloaded configuration $\mathcal{B}_{1}$, we assume zero net axial force and bending moment [12] and zero normal traction on the inner and outer boundaries,

$$
\begin{gathered}
\int_{\rho(a)}^{\rho(c)} t_{\zeta} \rho d \rho=0, \quad \int_{\rho(a)}^{\rho(c)} t_{\vartheta} \rho d \rho=0, \\
t_{\rho}[\rho(a)]=t_{\rho}[\rho(c)]=0,
\end{gathered}
$$

where $\rho(a)$ and $\rho(c)$ are the inner and outer radii in $\mathcal{B}_{1}$.

Substituting the expressions from (26) into the incompressibility condition (27) yields

$$
\frac{\pi \Lambda r}{\varphi_{0} \rho} \frac{\partial r}{\partial \rho}=1
$$

which can be integrated to obtain

$$
\rho(r)=\left(\rho(a)^{2}+\frac{\pi \Lambda}{\varphi_{0}}\left(r^{2}-a^{2}\right)\right)^{1 / 2} .
$$

Equations (30) can be written in terms of the material cooordinate $r$ by using the relationship in (32),

$$
\frac{\pi \Lambda}{\varphi_{0}} \int_{a}^{c} r t_{\zeta}(r) d r=0, \quad \frac{\pi \Lambda}{\varphi_{0 n h}} \int_{a}^{c} r t_{\vartheta}(r) d r=0 .
$$

The equilibrium equation (28) is integrated to obtain an equation for the radial stress

$$
t_{\rho}(r)=\frac{\pi \Lambda}{\varphi_{0}} \int_{a}^{c} \frac{r}{\rho(r)^{2}}\left(t_{\vartheta}(r)-t_{\rho}(r)\right) d r .
$$

Substituting the boundary condition at the outer boundary $\left(t_{\rho}(c)=0\right)$ into the previous equation yields

$$
\frac{\pi \Lambda}{\varphi_{0}} \int_{a}^{c}\left(\frac{r}{\rho(r)^{2}}\left(t_{\vartheta}(r)-t_{\rho}(r)\right) d r=0 .\right.
$$

Equations (34) and (36) are used to solve for the unknown parameters $\rho(a), \Lambda$, and $\varphi_{0}$ and the solution for $\mathcal{B}_{1}$ is completely determined. For the set of parameter values given above for a rabbit carotid artery, the opening angle was found to be $160^{\circ}$. Assuming axial growth is given by $\gamma_{z i}=1.2$ and $\gamma_{z o}=1$, the opening angle is plotted with respect to varying values of the radial growth. The radial growth corresponding to an opening angle of $160^{\circ}$ can then be determined (see Figure 5).

The computed stresses in the wall of both a pressurized artery with no residual stress and a pressurized artery with residual stress are shown in Figure 6. Note that the transmural stress gradient is greatly reduced when residual stress is included in the model. We recover here the well- known effect of residual stress on the stress field across the arterial wall [40].

\section{$4 \quad$ Stability Analysis}

\subsection{Incremental deformation}

We consider an incremental deformation superimposed on the finite deformation to perform the stability of the grown cylinder under applied loads. A deformation $\chi$ is introduced as

$$
\chi=\chi^{(0)}+\epsilon \chi^{(1)},
$$

where $\chi^{(0)}$ is the finite deformation, $\chi^{(1)}$ is the incremental deformation, and $\epsilon$ is a small parameter introduced to characterize the size of the perturbation. It follows that the total deformation tensor and the elastic tensor are given by

$$
\mathbf{F}=\operatorname{Grad}(\boldsymbol{\chi})=\left(\mathbf{1}+\epsilon \mathbf{F}^{(1)}\right) \cdot \mathbf{F}^{(0)},
$$




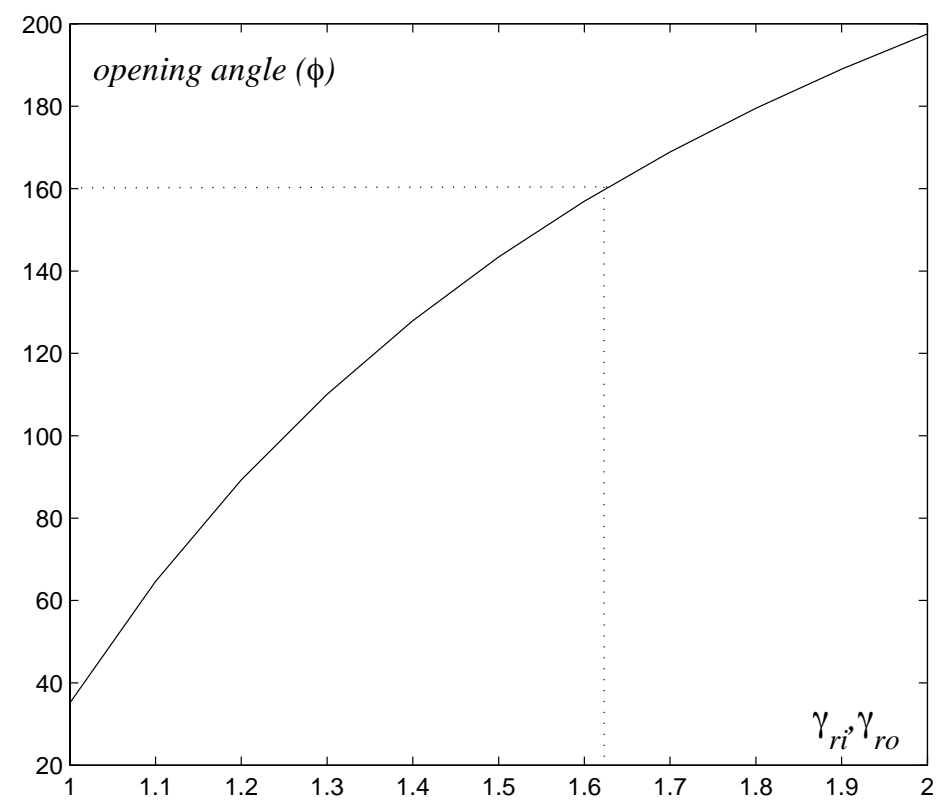

Figure 5: The opening angle of an artery due to a radial is plotted versus the radial growth. The axial growth is assumed to be $\gamma_{z i}=1.2, \gamma_{z o}=1$.

$$
\mathbf{A}=\left(\mathbf{1}+\epsilon \mathbf{A}^{(1)}\right) \cdot \mathbf{A}^{(0)},
$$

where $\mathbf{A}^{(0)}=\mathbf{F}^{(0)} \cdot \mathbf{G}^{-1}$ and $\mathbf{A}^{(1)}=\mathbf{F}^{(1)}$. The Cauchy stress tensor is expanded around the finite state in $\epsilon$ given by

$$
\mathbf{T}=\mathbf{T}^{(0)}+\epsilon \mathbf{T}^{(1)}+O\left(\epsilon^{2}\right),
$$

and the constitutive relationship to zeroth and first order reads

$$
\mathbf{T}^{(0)}=\mathbf{A}^{(0)} \cdot W_{\mathbf{A}}^{(0)}-p^{(0)} \mathbf{1},
$$

and

$$
\mathbf{T}^{(1)}=\mathcal{L}: \mathbf{F}^{(1)}+\mathbf{F}^{(1)} \cdot \mathbf{A}^{(0)} \cdot W_{\mathbf{A}}^{(0)}-p^{(1)} \mathbf{1}
$$

where $p=p^{(0)}+p^{(1)} \epsilon$, the derivatives of $W$ are with respect to $\mathbf{A}$ evaluated at $\mathbf{A}^{(0)}$, and $\mathcal{L}$ is the fourth-order tensor of instantaneous elastic moduli defined by

$$
\mathcal{L}: \mathbf{F}^{(1)}=\mathbf{A}^{(0)} \cdot W_{\mathbf{A A}}^{(0)}: \mathbf{F}^{(1)} \cdot \mathbf{A}^{(0)} .
$$

In components, the instantaneous elastic moduli are:

$$
\mathcal{L}_{i j k l}=A_{i m}^{(0)} \mathcal{A}_{m j k n} A_{n l}^{(0)},
$$

where $\mathcal{A}$ is the elastic moduli defined by $\mathcal{A}=\frac{\partial^{2} W}{\partial \mathbf{A} \partial \mathbf{A}}\left(\mathbf{A}^{(0)}\right)$. Since the potential is the sum of an isotropic and an anisotropic term, we decompose the elastic moduli in isotropic and anisotropic components

$$
\mathcal{L}_{i j k l}=\mathcal{L}_{i j k l}^{i s o}+\mathcal{L}_{i j k l}^{\text {aniso }}
$$

where the non-zero components of $\mathcal{L}^{\text {iso }}$ are given by [45]

$$
\begin{aligned}
& \mathcal{L}_{i i j j}^{i s o}=\mathcal{L}_{j j i i}^{i s o}=\frac{\alpha_{i} \alpha_{j}}{J} \frac{\partial^{2} W_{i s o}}{\partial \alpha_{i} \partial \alpha_{j}}, \\
& \mathcal{L}_{i j i j}^{i s o}=\alpha_{i}^{2} \frac{\alpha_{i} \frac{\partial W_{i s o}}{\partial \alpha_{i}}-\alpha_{j} \frac{\partial W_{i s o}}{\partial \alpha_{j}}}{\alpha_{i}^{2}-\alpha_{j}^{2}}, \quad i \neq j, \quad \alpha_{i} \neq \alpha_{j}, \\
& \mathcal{L}_{i j i j}^{i s o}-\mathcal{L}_{i j j i}^{i s o}=\mathcal{L}_{i j i j}^{i s o}-\mathcal{L}_{j i i j}^{i s o}=\alpha_{i} \frac{\partial W_{i s o}}{\partial \alpha_{i}}, \quad i \neq j .
\end{aligned}
$$


a)

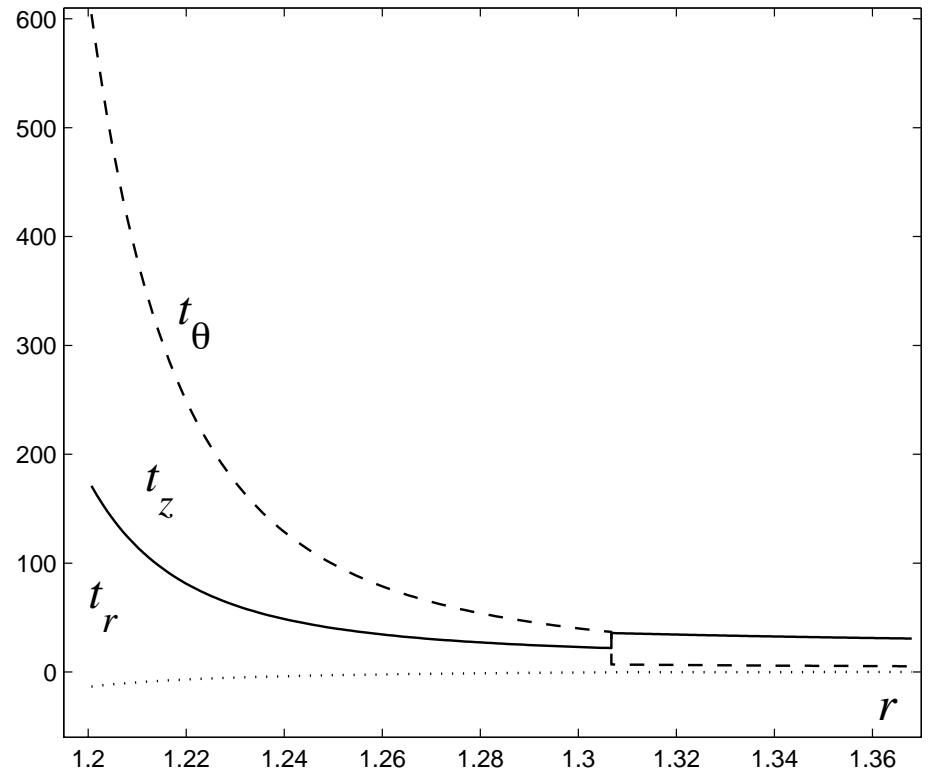

b)

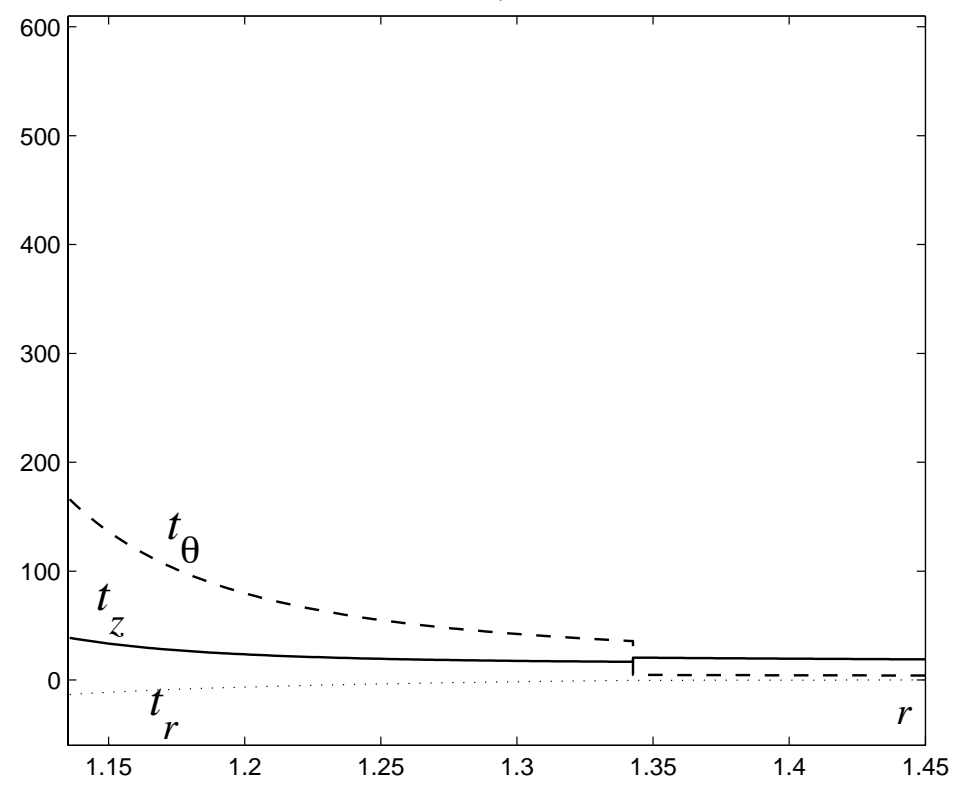

Figure 6: Stresses in the arterial wall a) without residual stress b) with residual stress. In both cases the artery is subjected to an internal pressure $P=13.33 \mathrm{kPa}$.

Since the above relations are ony valid for isotropic material, the coefficients of $\mathcal{L}_{i j k l}^{\text {aniso }}$ are given by another relationship (see [46]). For our particular problem, due to the particular dependence of the anisotropic part on $I_{4}$ and $I_{6}$ only, we have

$$
\begin{aligned}
\mathcal{L}_{\text {ijkl }}^{\text {aniso }}=\quad 2( & \frac{\partial W_{\text {aniso }}}{\partial I_{4}} m_{i} m_{k} \delta_{j l}+\frac{\partial W_{\text {aniso }}}{\partial I_{6}} m_{i}^{\prime} m_{k}^{\prime} \delta_{j l} \\
& \left.+2 \frac{\partial^{2} W_{\text {aniso }}}{\partial I_{4}^{2}} m_{i} m_{j} m_{k} m_{l}+2 \frac{\partial^{2} W_{\text {aniso }}}{\partial I_{6}^{2}} m_{i}^{\prime} m_{j}^{\prime} m_{k}^{\prime} m_{l}^{\prime}\right)
\end{aligned}
$$


where $m_{i}=\alpha_{i} M_{i}, m_{i}^{\prime}=\alpha_{i} M_{i}^{\prime}$ so explicitly $m_{1}=m_{1}^{\prime}=0, m_{2}=m_{2}^{\prime}=\alpha_{2} \cos \beta, m_{3}=-m_{3}^{\prime}=\alpha_{3} \sin \beta$. Note that there is no cross-derivatives w.r.t. $I_{4}$ and $I_{6}$ due to the fact that our particula potential does not contain terms that involve both invariants. We also have

$$
W_{4}=\frac{\partial W_{\text {aniso }}}{\partial I_{4}}=\frac{\partial W_{\text {aniso }}}{\partial I_{6}}=k_{1} e^{\nu\left(-1+\cos \beta^{2} \alpha_{2}^{2}+\sin \beta^{2} \alpha_{3}^{2}\right)^{2}}\left(-1+\cos \beta^{2} \alpha_{2}^{2}+\sin \beta^{2} \alpha_{3}^{2}\right)
$$

and

$$
\begin{aligned}
W_{44}= & \frac{\partial^{2} W_{\text {aniso }}}{\partial I_{4}^{2}}=\frac{\partial^{2} W_{\text {aniso }}}{\partial I_{6}^{2}}= \\
& k_{1} e^{\nu\left(-1+\cos \beta^{2} \alpha_{2}^{2}+\sin \beta^{2} \alpha_{3}^{2}\right)^{2}}\left[1+2 \nu\left(-1+\cos \beta^{2} \alpha_{2}^{2}+\sin \beta^{2} \alpha_{3}^{2}\right)^{2}\right]
\end{aligned}
$$

due to the fact that $I_{4}=I_{6}=\alpha_{2}^{2} \cos \beta^{2}+\alpha_{3}^{2} \sin \beta^{2}$. Since $m_{3}=-m_{3}^{\prime}$, the only non-zero coefficients are:

$$
\begin{aligned}
& \mathcal{L}_{2121}^{\text {aniso }}=4 W_{4} M_{2}^{2} \alpha_{2}^{2}, \\
& \mathcal{L}_{2222}^{\text {aniso }}=4 W_{4} M_{2}^{2} \alpha_{2}^{2}+8 W_{44} M_{2}^{4} \alpha_{2}^{4}, \\
& \mathcal{L}_{2233}^{\text {aniso }}=8 W_{44} M_{2}^{2} M_{3}^{2} \alpha_{2}^{2} \alpha_{3}^{2}, \\
& \mathcal{L}_{2323}^{\text {aniso }}=4 W_{4} M_{2}^{2} \alpha_{2}^{2}+8 W_{44} M_{2}^{2} M_{3}^{2} \alpha_{2}^{2} \alpha_{3}^{2}, \\
& \mathcal{L}_{2332}^{\text {aniso }}=8 W_{44} M_{2}^{2} M_{3}^{2} \alpha_{2}^{2} \alpha_{3}^{2}, \\
& \mathcal{L}_{3131}^{\text {aniso }}=4 W_{4} M_{3}^{2} \alpha_{3}^{2}, \\
& \mathcal{L}_{3223}^{\text {aniso }}=8 W_{44} M_{2}^{2} M_{3}^{2} \alpha_{2}^{2} \alpha_{3}^{2}, \\
& \mathcal{L}_{3232}^{\text {aniso }}=4 W_{4} M_{3}^{2} \alpha_{3}^{2}+8 W_{44} M_{2}^{2} M_{3}^{2} \alpha_{2}^{2} \alpha_{3}^{2}, \\
& \mathcal{L}_{3322}^{\text {aniso }}=8 W_{44} M_{2}^{2} M_{3}^{2} \alpha_{2}^{2} \alpha_{3}^{2}, \\
& \mathcal{L}_{3333}^{\text {aniso }}=4 W_{4} M_{3}^{2} \alpha_{3}^{2}+8 W_{44} M_{3}^{4} \alpha_{3}^{4} .
\end{aligned}
$$

The equilibrium equation to first order is

$$
\operatorname{div}\left(\mathbf{T}^{(1)}\right)=0
$$

where div is the divergence in the current configuration. Plugging (40) into (58) and using $\left(\mathbf{T}^{(i)}\right)^{\mathrm{T}}=\left(\mathbf{T}^{(i)}\right)$, $\operatorname{div}\left(\mathbf{T}^{(0)}\right)=0$ and $\operatorname{div}\left(\mathbf{F}^{(1)}\right)=0$, the incremental equations for an incompressible material are given by

$$
\operatorname{div}\left(\mathcal{L}: \mathbf{F}^{(1)}\right)+\left(\mathbf{F}^{(1)}\right)^{\mathrm{T}} \cdot \operatorname{grad}\left(p^{(0)}\right)-\operatorname{grad}\left(p^{(1)}\right)=0 .
$$

Explicitly, these three equations are given by

$$
\begin{gathered}
\frac{\partial \mathcal{M}_{11}}{\partial r}+\frac{1}{r} \frac{\partial \mathcal{M}_{21}}{\partial \theta}+\frac{\partial \mathcal{M}_{31}}{\partial z}+\frac{1}{r}\left(\mathcal{M}_{11}-\mathcal{M}_{22}\right)+F_{11}^{(1)} \frac{d p^{(0)}}{d r}-\frac{\partial p^{(1)}}{\partial r}=0, \\
\frac{\partial \mathcal{M}_{12}}{\partial r}+\frac{1}{r} \frac{\partial \mathcal{M}_{22}}{\partial \theta}+\frac{\partial \mathcal{M}_{32}}{\partial z}+\frac{1}{r}\left(\mathcal{M}_{21}+\mathcal{M}_{12}\right)+F_{12}^{(1)} \frac{d p^{(0)}}{d r}-\frac{\partial p^{(1)}}{\partial \theta}=0, \\
\frac{\partial \mathcal{M}_{13}}{\partial r}+\frac{1}{r} \frac{\partial \mathcal{M}_{23}}{\partial \theta}+\frac{\partial \mathcal{M}_{33}}{\partial z}+\frac{1}{r} \mathcal{M}_{13}+F_{13}^{(1)} \frac{d p^{(0)}}{d r}-\frac{\partial p^{(1)}}{\partial z}=0,
\end{gathered}
$$

where $\mathcal{M}=\mathcal{L}: \mathbf{F}^{(1)}$.

Assuming that no extra loading is imposed on the surface of the cylinder, the incremental boundary conditions are given by $\left(\mathbf{S}^{(1)}\right)^{\mathrm{T}} \cdot \mathbf{N}=0$, where $\mathbf{S}^{(1)}$ is the increment in the nominal stress referred to the current configuration. For given boundary conditions, bifurcation occurs if there exists a non-trivial solution to the system of equations given by (60)-(62). 


\subsection{Bifurcation analysis}

The general form of the incremental deformation is given by the vector

$$
\chi^{(1)}=[u(r, \theta, z), v(r, \theta, z), w(r, \theta, z)] .
$$

The incremental deformation tensor $\mathbf{F}^{(1)}$ is then given by

$$
\mathbf{F}^{(1)}=\left[\begin{array}{ccc}
u_{r} & \left(u_{\theta}-v\right) / r & u_{z} \\
v_{r} & \left(u+v_{\theta}\right) / r & v_{z} \\
w_{r} & w_{\theta} / r & w_{z}
\end{array}\right],
$$

where $(r, \theta, z)$ subscripts denote partial derivatives. The incompressibility constraint is

$$
\operatorname{tr}\left(\mathbf{F}^{(1)}\right) \equiv u_{r}+\left(u+v_{\theta}\right) / r+w_{z}=0 .
$$

Substituting (64) into the incremental equations given in (59) results in

$$
\begin{gathered}
\left(r\left(\mathcal{L}_{2211}^{\prime}-\mathcal{L}_{3311}^{\prime}\right)-\mathcal{L}_{2222}+\mathcal{L}_{2233}-\mathcal{L}_{2121}+\mathcal{L}_{1331}\right) v_{\theta} / r^{2}+\left(\mathcal{L}_{1111}-\mathcal{L}_{3311}-\mathcal{L}_{1331}\right) u_{r r} \\
+\left(\mathcal{L}_{2112}+\mathcal{L}_{2211}-\mathcal{L}_{1331}-\mathcal{L}_{3311}\right) v_{r \theta} / r+\mathcal{L}_{2121} u_{\theta \theta} / r^{2}+\mathcal{L}_{3131} \\
+\left(r\left(\mathcal{L}_{1111}^{\prime}+\left(p^{(0)}\right)^{\prime}-\mathcal{L}_{3311}^{\prime}\right)+\mathcal{L}_{1111}-2 \mathcal{L}_{3311}+\mathcal{L}_{2233}-\mathcal{L}_{1331}\right) u_{r} / r \\
+\left(r\left(\mathcal{L}_{2211}^{\prime}-\mathcal{L}_{3311}^{\prime}\right)-\mathcal{L}_{2222}+\mathcal{L}_{2233}+\mathcal{L}_{1331}\right) u / r^{2}-\left(p^{(1)}\right)_{r}=0 \\
\left(r \mathcal{L}_{1212}^{\prime}+\mathcal{L}_{1212}+\mathcal{L}_{2222}-\mathcal{L}_{2233}-\mathcal{L}_{3223}\right) u_{\theta} / r-\left(r \mathcal{L}_{1212}^{\prime}+\mathcal{L}_{1212}\right) v / r \\
\quad+\left(\mathcal{L}_{2222}-\mathcal{L}_{2233}-\mathcal{L}_{3223}\right) v_{\theta \theta} / r+\mathcal{L}_{3232} r v_{z z}+\mathcal{L}_{1212} r v_{r r} \\
+\left(\mathcal{L}_{2211}-\mathcal{L}_{2233}-\mathcal{L}_{3223}+\mathcal{L}_{1221}\right) u_{r \theta}+\left(r \mathcal{L}_{1212}^{\prime}+\mathcal{L}_{1212}\right) v_{r}-\left(p^{(1)}\right)_{\theta}=0 \\
\mathcal{L}_{2323} w_{\theta \theta} / r^{2}+\left(r \mathcal{L}_{1313}^{\prime}+r\left(p^{(0)}\right)^{\prime}+\mathcal{L}_{1331}-\mathcal{L}_{2332}\right) u_{z} / r \\
+\mathcal{L}_{1313} w_{r r}+\left(\mathcal{L}_{3333}-\mathcal{L}_{2332}-\mathcal{L}_{2233}\right) w_{z z}+\left(r \mathcal{L}_{1313}^{\prime}+\mathcal{L}_{1313}\right) w_{r} / r \\
\quad+\left(\mathcal{L}_{3311}+\mathcal{L}_{1331}-\mathcal{L}_{2332}-\mathcal{L}_{2233}\right) u_{r z}-\left(p^{(1)}\right)_{z}=0
\end{gathered}
$$

where the prime denotes differentiation with respect to $r$. The set of partial differential equations for the variables $u, v, w, p^{(1)}$ can be further simplified to a boundary value problem for a set of differential equations in the variable $r$ by Fourier expanding the dependence in $\theta$ and $z$, that is:

$$
\begin{aligned}
u & =f(r) \cos m \theta \cos \psi z, \\
v & =g(r) \sin m \theta \cos \psi z, \\
w & =h(r) \cos m \theta \sin \psi z, \\
p^{(1)} & =k(r) \cos m \theta \cos \psi z .
\end{aligned}
$$

In order that the incremental displacements be single-valued, we take the mode number $m$ to be an integer $m \geq 0$. Since the incremental end displacement $w$ must vanish on the ends, we have

$$
\psi=n \pi / l=n \pi /(\lambda L), \quad n=1,2,3, \ldots
$$

where $n$ is set to unity so that $\psi$ is inversely proportional to the length of the cylinder. The expressions in (72) are substituted in (66) - (68) and $h(r)$ can be eliminated by means of the incompressibility constraint (65). The resulting incremental equilibrium equations are

$$
\begin{aligned}
k^{\prime}= & \left(r\left(\mathcal{L}_{2211}^{\prime}-\mathcal{L}_{3311}^{\prime}\right)-\mathcal{L}_{2222}+\mathcal{L}_{2233}-\mathcal{L}_{2121}+\mathcal{L}_{1331}\right) m g / r^{2} \\
& +\left(\mathcal{L}_{2112}+\mathcal{L}_{2211}-\mathcal{L}_{1331}-\mathcal{L}_{3311}\right) m g^{\prime} / r+\left(\mathcal{L}_{1111}-\mathcal{L}_{3311}-\mathcal{L}_{1331}\right) f^{\prime \prime}
\end{aligned}
$$




$$
\begin{aligned}
& +\left(r\left(\mathcal{L}_{1111}^{\prime}+\left(p^{(0)}\right)^{\prime}-\mathcal{L}_{3311}^{\prime}\right)+\mathcal{L}_{1111}-2 \mathcal{L}_{3311}+\mathcal{L}_{2233}-\mathcal{L}_{1331}\right) f^{\prime} / r \\
& +\left(r\left(\mathcal{L}_{2211}^{\prime}-\mathcal{L}_{3311}^{\prime}\right)-\mathcal{L}_{2222}+\mathcal{L}_{2233}+\mathcal{L}_{1331}\right) f / r^{2}-m^{2} \mathcal{L}_{2121} f / r^{2}-\psi^{2} \mathcal{L}_{3131} f \\
& r \mathcal{L}_{1212} g^{\prime \prime}=-m k+\left(r \mathcal{L}_{1212}^{\prime}+\mathcal{L}_{1212}+\mathcal{L}_{2222}-\mathcal{L}_{2233}-\mathcal{L}_{3223}\right) m f / r \\
& \quad+\left(r \mathcal{L}_{1212}^{\prime}+\mathcal{L}_{1212}\right) g / r+\left(\mathcal{L}_{2222}-\mathcal{L}_{2233}-\mathcal{L}_{3223}\right) m^{2} g / r \\
& +r \psi^{2} \mathcal{L}_{3232} g+\left(\mathcal{L}_{2211}-\mathcal{L}_{2233}-\mathcal{L}_{3223}+\mathcal{L}_{1221}\right) m f^{\prime}-\left(r \mathcal{L}_{1212}^{\prime}+\mathcal{L}_{1212}\right) g^{\prime} \\
& \mathcal{L}_{1313} f^{\prime \prime \prime}=m^{2} f^{\prime}+(m g+f) / r \mathcal{L}_{2323} / r^{2}-\left(r \mathcal{L}_{1313}^{\prime}+r\left(p^{(0)}\right)^{\prime}+\mathcal{L}_{1331}-\mathcal{L}_{2332}\right) \psi^{2} f / r \\
& \quad-\mathcal{L}_{1313}\left(\left(f^{\prime \prime}+m g^{\prime \prime}\right) / r-2\left(r^{\prime}+m g^{\prime}\right) / r^{2}+2(f+m g) / r^{3}\right) \\
& +\left(\mathcal{L}_{3333}-\mathcal{L}_{2332}-\mathcal{L}_{2233}\right) \psi^{2}(f+m g) / r+\left(\mathcal{L}_{3333}+\mathcal{L}_{3311}-\mathcal{L}_{1331}\right) \psi^{2} f^{\prime} \\
& -\left(r \mathcal{L}_{1313}^{\prime}+\mathcal{L}_{1313}\right)\left(f^{\prime \prime}+\left(f^{\prime}+m g^{\prime}\right) / r-(f+m g) / r^{2}\right) / r-\psi^{2} k
\end{aligned}
$$

The corresponding incremental boundary condition on the inner and outer wall $r=a, c$ is $\mathbf{T}^{(1)} \cdot \mathbf{n}=P^{(1)} \mathbf{n}$ which takes the form

$$
\mathbf{T}^{(1)} \cdot \mathbf{n}=\left(\mathcal{L}: \mathbf{F}^{(1)}+\mathbf{F}^{(1)} \mathbf{A}^{(0)} \cdot W_{\mathbf{A}}^{(0)}-p^{(1)} \mathbf{1}\right) \cdot \mathbf{n}=\mathbf{0},
$$

where the incremental internal pressure, $P^{(1)}$, is taken to be $P^{(1)}=0$. The three boundary condition equations on $r=a, c$ are given by

$$
\begin{gathered}
\left(\mathcal{L}_{1122}-\mathcal{L}_{1133}\right)(f+m g)+\left(\mathcal{L}_{1111}-\mathcal{L}_{1133}+\alpha_{r} W_{1}\right) r f^{\prime}-r k=0, \quad r=a, c, \\
r g^{\prime}-g-m f=0, \quad r=a, c, \\
r^{2} f^{\prime \prime}+r f^{\prime}+\left(\psi^{2} r^{2}+m^{2}-1\right) f=0, \quad r=a, c .
\end{gathered}
$$

One must now consider the boundary conditions at the interface of the two layers. It is assumed that there is no penetration and no gaps nor slippage at the interface and therefore, $f_{1}=f_{2}, g_{1}=g_{2}$, and $h_{1}=h_{2}$ where the subscripts 1,2 correspond to the inner layer and outer layer, respectively. Recall that $h$ can be eliminated by using the incompressible condition in (65) and therefore the boundary condition $h_{1}=h_{2}$ is equivalent to requiring $f_{1}^{\prime}=f_{2}^{\prime}$. Lastly, the stresses at the interface are required to be continuous, i.e. the bond is rigid. The rest of the boundary conditions are as follows:

$$
\begin{gathered}
\left(\mathcal{L}_{1122}^{(1)}-\mathcal{L}_{1133}^{(1)}\right)\left(f_{1}+m g_{1}\right)+\left(\mathcal{L}_{1111}^{(1)}-\mathcal{L}_{1133}^{(1)}+\alpha_{r} \frac{\partial W}{\partial \alpha_{r}}\right) f_{1}^{\prime} r-r k_{1} \\
=\left(\mathcal{L}_{1122}^{(2)}-\mathcal{L}_{1133}^{(2)}\right)\left(f_{2}+m g_{2}\right)+\left(\mathcal{L}_{1111}^{(2)}-\mathcal{L}_{1133}^{(2)}+\alpha_{r} \frac{\partial W}{\partial \alpha_{r}}\right) f_{2}^{\prime} r-r k_{2}, \quad r=b, \\
\mathcal{L}_{1212}^{(1)}\left(r g_{1}^{\prime}-m f_{1}-g_{1}\right)=\mathcal{L}_{1212}^{(2)}\left(r g_{2}^{\prime}-m f_{2}-g_{2}\right), \quad r=b, \\
\mathcal{L}_{1313}^{(1)}\left(r^{2} f_{1}^{\prime \prime}+r f_{1}^{\prime}+\left(\psi^{2} r^{2}-1\right) f_{1}+r m g_{1}^{\prime}-m g_{1}\right) \\
=\mathcal{L}_{1313}^{(2)}\left(r^{2} f_{2}^{\prime \prime}+r f_{2}^{\prime}+\left(\psi^{2} r^{2}-1\right) f_{2}+r m g_{2}^{\prime}-m g_{2}\right), \quad r=b, \\
f_{1}=f_{2}, \quad r=b, \\
f_{1}^{\prime}=f_{2}^{\prime}, \quad r=b, \\
g_{1}=g_{2}, \quad r=b,
\end{gathered}
$$

where the superscripts (1) and (2) correspond to the inner and outer layers, respectively. The growth dependence comes about through the coefficients of the above system of equations. The instantaneous elastic moduli depend on $\alpha$ and its derivatives through the relation

$$
\frac{d \alpha}{d r}=\frac{\alpha}{r}-\frac{\lambda_{z} \alpha^{3} \gamma_{\theta}^{2}}{r \gamma}-\frac{\alpha \gamma_{\theta}^{\prime}}{\gamma_{\theta}}
$$




\subsection{Numerical Integration}

The coupled equations for $f(r), g(r)$, and $k(r)$ can be written in the form

$$
\mathbf{y}^{\prime}=\mathbf{f}(\mathbf{y}, r), \quad \mathbf{y} \in \Re^{6},
$$

where $\mathbf{y}=\left(f, f^{\prime}, f^{\prime \prime}, g, g^{\prime}, k\right)$. The boundary conditions (78)-(86) are given by a set of twelve linear functions $c_{1, \ldots, 12}(\mathbf{y}(r) ; r)$,

$$
\begin{gathered}
c_{1,2,3}\left(\mathbf{y}_{1}(a) ; a\right)=0, \quad c_{4,5,6}\left(\mathbf{y}_{2}(c) ; c\right)=0, \\
c_{7,8,9,10,11,12}\left(\mathbf{y}_{1}(b) ; b\right)=c_{7,8,9,10,11,12}\left(\mathbf{y}_{2}(b) ; b\right) .
\end{gathered}
$$

The determinant method $[47,35,48]$ is used in order to find values of the parameters for which a solution exists. Consider three copies of the system (88), that is

$$
\mathbf{y}_{1}^{(1)^{\prime}}=\mathbf{f}_{1}\left(\mathbf{y}_{1}^{(1)} ; r\right), \quad \mathbf{y}_{1}^{(2)^{\prime}}=\mathbf{f}_{1}\left(\mathbf{y}_{1}^{(2)} ; r\right), \quad \mathbf{y}_{1}^{(3)^{\prime}}=\mathbf{f}_{1}\left(\mathbf{y}_{1}^{(3)} ; r\right)
$$

with linearly independent conditions $\mathbf{y}_{1}^{(1)}(a), \mathbf{y}_{1}^{(2)}(a), \mathbf{y}_{1}^{(3)}(a)$ that satisfy the conditions at $r=a$ such that $c_{1,2,3}\left(\mathbf{y}_{1}^{(1,2,3)}(a) ; a\right)=0$. Integrate this system up to $r=b$, then obtain the initial conditions $\mathbf{y}_{2}^{(1)}(b), \mathbf{y}_{2}^{(2)}(b)$, $\mathbf{y}_{2}^{(3)}(b)$ from the end conditions $\mathbf{y}_{1}^{(1)}(b), \mathbf{y}_{1}^{(2)}(b), \mathbf{y}_{1}^{(3)}(b)$ using the boundary conditions at the interface in Equation (90). Integrate this system up to $r=c$, then evaluate the $3 \times 3$ determinant at $r=c$ given by

$$
\Delta(r)=\left|\begin{array}{ccc}
c_{4}\left(\mathbf{y}_{2}^{(1)}(r) ; r\right) & c_{4}\left(\mathbf{y}_{2}^{(1)}(r) ; r\right) & c_{4}\left(\mathbf{y}_{2}^{(1)}(r) ; r\right) \\
c_{5}\left(\mathbf{y}_{2}^{(2)}(r) ; r\right) & c_{5}\left(\mathbf{y}_{2}^{(2)}(r) ; r\right) & c_{5}\left(\mathbf{y}_{2}^{(2)}(r) ; r\right) \\
c_{6}\left(\mathbf{y}_{2}^{(3)}(r) ; r\right) & c_{6}\left(\mathbf{y}_{2}^{(3)}(r) ; r\right) & c_{6}\left(\mathbf{y}_{2}^{(3)}(r) ; r\right)
\end{array}\right|
$$

Bifurcation occurs when there is a linear combination of $y_{2}^{(1)}, y_{2}^{(2)}$, and $y_{2}^{(3)}$ that satisfies the system (88) with boundary conditions (89) and (90). For some initial length of the cylinder, we iterate on $\lambda_{z}$ until the determinant vanishes.

\section{Results}

\subsection{Inversion lines}

In order to study the mechanical factors responsible for the constancy of artery length in normal physiological regime, we consider the pressure/axial stretch plot in Figure 7 with parameters from [43] and no residual stress. An interesting trend is revealed. As the external load is increased, the length does not change as the pressure increases which agrees with the experimental findings where the end-to-end distance of major arteries remained mostly unchanged despite large variations of pressure [1, 2]. This result is consistent with the inversion line found in [49] and attributed to the anisotropic response of the material. However, the pressure is plotted with respect to the axial stretch in Figure 8 for a neo-Hookean material, isotropic Fung material, and anisotropic Fung material, and the inversion lines appear in both the isotropic and anisotropic Fung cylinders. The neo-Hookean cylinder does not exhibit inversion lines, but instead reveals a local maximum which is associated with the well-known limit-point instability. Therefore, the inversion lines are not due to the anisotropy property of the material or residual stress but instead due to the exponential nonlinearity as modeled by the Fung material (See also [50] for similar results).

\subsection{Buckling}

A bifurcation analysis can now be used to determine the critical buckling pressure and to study the overall effect of axial stretch and residual stress on the critical pressure. The Holzapfel model is used with the parameter values defined in Section 2.4. It should be noted that the parameter values $a_{0}, c_{0}, l_{0}$ are the geometric parameters of the grown cylinder. The grown cylinder is then subjected to an internal pressure and axial stretch, and the resulting cylinder has an inner radius $a$, outer radius $c$, and length $l$. The axial stretch $\lambda$ refers to the ratio $l / l_{0}$. The artery is first subjected to a constant axial stretch, and then the internal pressure is increased until the artery buckles. The critical internal buckling pressure and axial stretch are plotted in Figure 9 for various values of axial growth. The stability curve for a grown residually stressed 


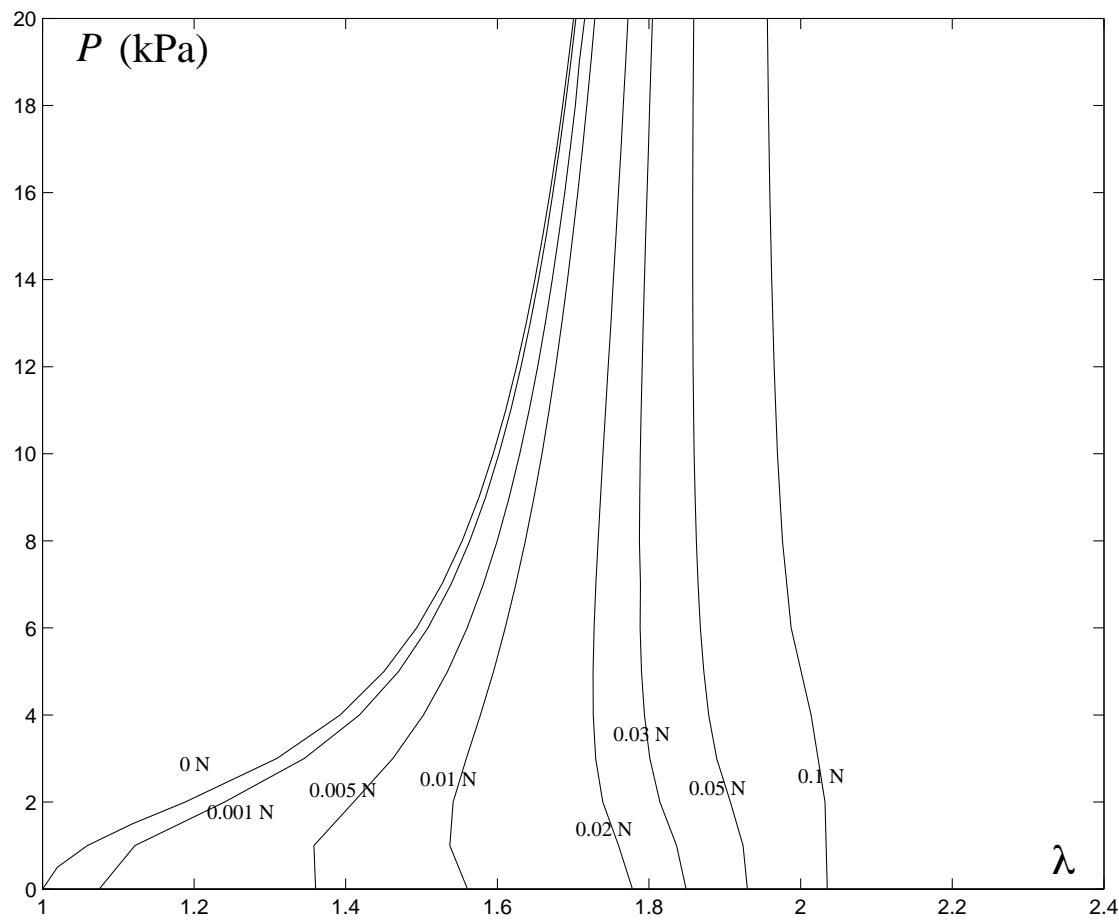

Figure 7: Pressure/axial stretch plot for a two-layer anisotropic cylinder without residual stress. Parameter values taken from Holzapfel [43].

cylinder is compared to a stress-free cylinder with the same geometrical parameters. Axial in situ stretches of 1.5 have been reported for rabbit iliac arteries [51]. Note that in this regime the residually stressed cylinder can withstand a much greater internal pressure before it buckles. Furthermore, an artery under a typical physiological internal pressure $P=13.33 \mathrm{kPa}$ is shown to withstand a greater reduced axial stretch if the artery is residually stressed.

Jackson et al. [22] performed a study that assessed the effect of reducing axial stretch in rabbit carotid arteries from $62 \pm 2 \%$ to $33 \pm 2 \%$ using interposition grafts. The arteries were held at an axial stretch of $30 \%$ for 12 weeks. The arteries displayed tissue growth and remodeling and all vessels became tortuous. Remarkably, our model predicts that an artery subjected to an internal pressure of $P=13.33 \mathrm{kPa}$ will buckle when the axial stretch is reduced to a value in the range between 1.25 and 1.33 for the given growth parameters (see Figure 9). We conclude that the onset of axial tortuosity in arteries is consistent with a buckling instability triggered by variations of internal pressure.

\section{Conclusion}

In this paper, we have considered a general mechanical model of arteries based on the model of Holzapfel et al. [13] for the mechanical part and the approach of Taber [34] for the modeling of residual stress. Whereas many studies have focused on the role of residual stress in the radial and hoop directions, the emphasis here was on the mechanical role of axial residual stress. The stability analysis reveals that for typical physiological values the axial residual stress plays an important role in preventing buckling. This observation is consistent with our analysis of rigidification of plant stems [52] which revealed the interplay between the nonlinear response of the material with the residual stress induced by differential growth. In the case where the arteries become unstable under the internal pressure through a buckling instability, the mechanical analysis of arteries presented here is consistent with the onset of such an instability. However, this analysis cannot 
explain the remodeling of the structure after the instability that will eventually lead to permanent tortuosity. Such an analysis would require either a numerical investigation based on mixture theory or the modeling of the arteries as an elastic tube or rods undergoing growth and remodeling.

Acknowledgments: This publication is based on work supported by Award No. KUK-C1-013-04, made by King Abdullah University of Science and Technology (KAUST), and based in part upon work supported by the National Science Foundation under grants DMS-0907773 (AG). The authors have also benefited from discussion with Michel Destrade.

\section{References}

[1] D. J. Patel and D. L. Fry. Longitudinal tethering of arteries in dogs. Circulation Research, 19(6):1011$1021,1966$.

[2] R. H. Cox. Anisotropic properties of the canine carotid artery in vitro. Journal of biomechanics, $8(5): 293,1975$.

[3] B. M. Learoyd and M. G. Taylor. Alterations with age in the viscoelastic properties of human arterial walls. Circulation Research, 18(3):278-292, 1966.

[4] L. Cardamone, A. Valentín, J. F. Eberth, and J. D. Humphrey. Origin of axial prestretch and residual stress in arteries. Biomechanics and modeling in mechanobiology, 2009.

[5] Y. C. Fung. What principle governs the stress distribution in living organs? In Y. C. Fung, E. Fukada, and J. J. Wang, editors, Biomechanics in China, Japan and USA, pages 1-13. Science Press, Beijing, 1983.

[6] R. N. Vaishnav and J. Vossoughi. Estimation of residual strains in aortic segments. In C. W. Hall, editor, Biomedical Engineering II: Recent Developments: Proceedings of the Second Southern Biomedical Engineering Conference, pages 330-333. Pergamon Press, NY, 1983.

[7] R. N. Vaishnav and J. Vossoughi. Residual stress and strain in aortic segments. J. Biomech., 20(3):235$239,1987$.

[8] Y. C. Fung. What are the residual stresses doing in our blood vessels? Annals of Biomedical engineering, 19(3):237-249, 1991.

[9] Y. C. Fung. Biomechanics: mechanical properties of living tissues. Springer, New York, 1993.

[10] H. R. Chaudhry, B. Bukiet, A. Davis, A. B. Ritter, and T. Findley. Residual stresses in oscillating thoracic arteries reduce circumferential stresses and stress gradients. Journal of biomechanics, 30(1):57$62,1997$.

[11] J. D. Humphrey. Cardiovascular solid mechanics. Cells, tissues, and organs. Springer Verlag, New York, 2002.

[12] A. Rachev. Theoretical study of the effect of stress-dependent remodeling on arterial geometry under hypertensive conditions. J. Biomech., 30:819-827, 1997.

[13] G. A. Holzapfel, T. C. Gasser, and R. W. Ogden. A new constitutive framework for arterial wall mechanics and a compartaive study of material models. J. Elasticity, 61:1-48, 2000.

[14] G. A. Holzapfel, M. Stadler, and C. A. J. Schulze-Bauer. A layer-specific three-dimensional model for the simulation of balloon angioplasty using magnetic resonance imaging and mechanical testing. Annals of Biomedical Engineering, 30(6):753-767, 2002.

[15] G.A. Holzapfel and R.W. Ogden. Modelling the layer-specific three-dimensional residual stresses in arteries, with an application to the human aorta. Journal of the Royal Society Interface, 2009. 
[16] L.A. Taber. An optimization principle for vascular radius including the effects of smooth muscle tone. Biophysical journal, 74(1):109-114, 1998.

[17] S. Lehoux, Y. Castier, and A. Tedgui. Molecular mechanisms of the vascular responses to haemodynamic forces. Journal of internal medicine, 259(4):381, 2006.

[18] B. H. Wayman, W. R. Taylor, A. Rachev, and R. P. Vito. Arteries respond to independent control of circumferential and shear stress in organ culture. Annals of Biomedical Engineering, 36(5):673-684, 2008.

[19] N. P. Davis, H. C. Han, B. Wayman, and R. Vito. Sustained axial loading lengthens arteries in organ culture. Annals of biomedical engineering, 33(7):867-877, 2005.

[20] Z.S. Jackson, A.I. Gotlieb, and B.L. Langille. Wall Tissue Remodeling Regulates Longitudinal Tension in Arteries. Circulation Research, 90(8):918-925, 2002.

[21] R. L. Gleason and J. D. Humphrey. Effects of a sustained extension on arterial growth and remodeling: a theoretical study. Journal of biomechanics, 38(6):1255-1261, 2005.

[22] Z. S. Jackson, D. Dajnowiec, A. I. Gotlieb, and B. L. Langille. Partial Off-Loading of Longitudinal Tension Induces Arterial Tortuosity. Arteriosclerosis, Thrombosis, and Vascular Biology, 25(5):957$962,2005$.

[23] D. M. Danks, P. E. Campbell, B. J. Stevens, V. Mayne, and E. Cartwright. Menkes's kinky hair syndrome; An inherited defect in copper absorption with widespread effects. Pediatrics, 50(2):188-201, 1972.

[24] P. Coyle. Diameter and length changes in cerebral collaterals after middle cerebral artery occlusion in the young rat. The Anatomical Record, 210(2):357 - 364, 1984.

[25] L.C. Ades, W.B. Knight, R.W. Byard, J.F. Bateman, J.A.D. Esquivel, R.B.B. Mee, E.A. Haan, and D.M. Milewicz. Clinicopathologic findings in congenital aneurysms of the great vessels. American journal of medical genetics, 66(3):289-299, 1996.

[26] P. Franceschini, A. Guala, D. Licata, G. Di Cara, and D. Franceschini. Arterial tortuosity syndrome. American journal of medical genetics, 91(2):141-143, 2000.

[27] R. Gardella, N. Zoppi, D. Assanelli, M.L. Muiesan, S. Barlati, and M. Colombi. Exclusion of candidate genes in a family with arterial tortuosity syndrome. American Journal of Medical Genetics, $126 \mathrm{~A}(3): 221-$ $228,2004$.

[28] E. Bullitt, M. G. Ewend, S. Aylward, W. Lin, G. Gerig, S. Joshi, I. Jung, K. Muller, and J. K. Smith. Abnormal vessel tortuosity as a marker of treatment response of malignant gliomas: preliminary report. Technol Cancer Res Treat, 3(6):577-584, Dec 2004.

[29] J. Weibel and W. S. Fields. Tortuosity, kinking and coiling of the internal carotid artery. Neurology, 15:7-11, 1965.

[30] P. Pancera, M. Ribul, B. Presciuttini, and A. Lechi. Prevalence of carotid artery kinking in 590 consecutive subjects evaluated by Echocolordoppler. Is there a correlation with arterial hypertension? Journal of Internal Medicine, 248(1):7, 2000.

[31] M. Aleksic, G. Schütz, S. Gerth, and J. Mulch. Surgical approach to kinking and coiling of the internal carotid artery. Journal of Cardiovascular Surgery, 45(1):43-48, 2004.

[32] H. C. Han. A biomechanical model of artery buckling. J. Biomech., 40:3672-3678, 2007.

[33] H. C. Han. Nonlinear buckling of blood vessels: A theoretical study. Journal of Biomechanics, 41(12):2708-2713, 2008.

[34] L. A. Taber. Biomechanical growth laws for muscle tissues. J. Theor. Biol., 193:201-213, 1998. 
[35] M. Ben Amar and A. Goriely. Growth and instability in elastic tissues. J. Mech. Phys. Solids, 53:2284$2319,2005$.

[36] E. K. Rodriguez, A. Hoger, and A. McCulloch. Stress-dependent finite growth in soft elastic tissue. J. Biomechanics, 27:455-467, 1994.

[37] G. A. Holzapfel, T. C. Gasser, and R. W. Ogden. Comparison of a multi-layer structural model for arterial walls with a Fung-type model, and issues of material stability. J. Biomech. Engineering, 126:264, 2004.

[38] P. Kalita and R. Schaefer. Mechanical Models of Artery Walls. Archives of Computational Methods in Engineering, 15(1):1-36, 2008.

[39] A. Delfino, N. Stergiopulos, J. E. Moore, and J. J. Meister. Residual strain effects on the stress field in a thick wall finite element model of the human carotid bifurcation. J. Biomech., 30(8):777-786, 1997.

[40] C. J. Chuong and Y. C. Fung. Three-dimensional stress distribution in arteries. Journal of biomechanical engineering, 105:268, 1983.

[41] R. N. Vaishnav and J. Vossoughi. Distribution of stresses and of strain-energy density through the wall thickness in a canine aortic segment. Circulation Research, 32:577, 1973.

[42] T. I. Baskin. Anisotropic expansion of the plant cell wall. Annu. Rev. Cell Dev. Biol., 21:203-222, 2005.

[43] G. A. Holzapfel. Computational biomechanics of soft biological tissue. Encyclopedia of computational mechanics, 2:605-635, 1984.

[44] L. A. Taber and J. D. Humphrey. Stress-modulated growth, residual stress, and vascular heterogeneity. J. Biomech. Eng., 123:528-535, 2001.

[45] R. W. Ogden. Non-linear elastic deformation. Dover, New york, 1984.

[46] D.A. Prikazchikov and G.A. Rogerson. On surface wave propagation in incompressible, transversely isotropic, pre-stressed elastic half-spaces. International Journal of Engineering Science, 42(10):967986, 2004.

[47] D. M. Haughton and A. Orr. On the eversion of incompressible elastic cylinders. Int. J. Non-Linear Mechanics, 30:81-95, 1995.

[48] D. M. Haughton and R. W. Ogden. Bifurcation of inflated circular cylinders of elastic material under axial loading-I. Membrane theory for thin-walled tubes. J. Mech. Phys. Solids, 27:179-212, 1979.

[49] C.A.J. Schulze-Bauer, C. Mörth, and G. A. Holzapfel. Passive biaxial mechanical response of aged human iliac arteries. J. Biomech. Engineering, 125:395, 2003.

[50] RW Ogden and CAJ Schulze-Bauer. Phenomenological and structural aspects of the mechanical response of arteries. ASME Applied Mech., 242:125-140, 2000.

[51] Y. C. Fung, K. Fronek, and P. Patitucci. Pseudoelasticity of arteries and the choice of its mathematical expression. American Journal of Physiology- Heart and Circulatory Physiology, 237(5):620-631, 1979.

[52] R. Vandiver and A. Goriely. Tissue tension and axial growth of cylindrical structures in plants and elastic tissues. Europhys. Lett. (EPL), 84(58004), 2008. 
a)
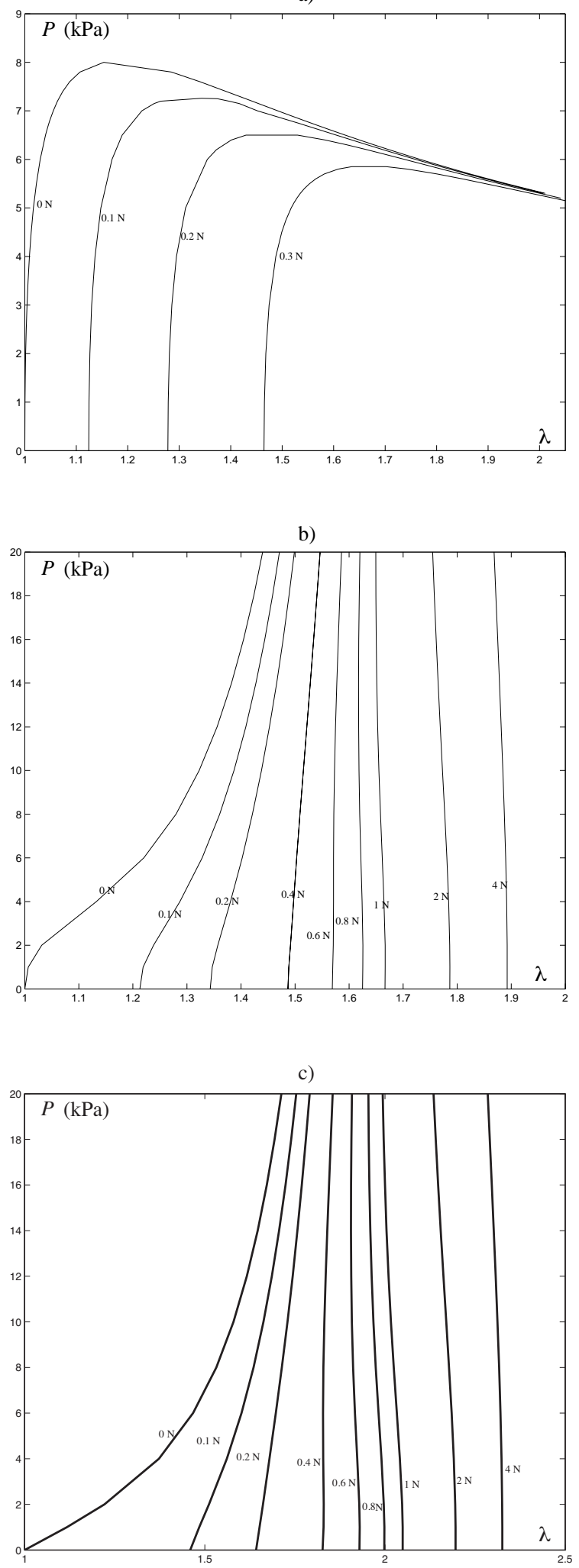

Figure 8: Pressure/axial stretch plot for a one-layer cylinder, $A=1.75, C=2.5$ without axial stress for $a$ ) a neo-Hookean material $\left(W=\frac{\mu}{2}\left(I_{1}-3\right)\right.$ where $\left.\left.\mu=30\right), b\right)$ an isotropic Fung material $\left(\mu=26.950, b_{1}=\right.$ $\left.\left.0.9925, b_{2}=0.418, b_{3}=0.0089, b_{4}=0.0749, b_{5}=0.0295, b_{6}=0.0193\right), c\right)$ an anisotropic material $\left(b_{1}=\right.$ $0.9925, b_{2}=0.418, b_{3}=0.0089, b_{4}=b_{5}=b_{6}=0$ ) (experiment number 71, [37]), where the Fung material is described by the strain-energy function $W=\frac{\mu}{2}\left(\exp _{19}(Q)-1\right)$ where $Q=b_{1} E_{\Theta \Theta}^{2}+b_{2} E_{Z Z}^{2}+b_{3} E_{R R}^{2}+$
$2 b_{4} E_{\Theta \Theta} E_{Z Z}+2 b_{5} E_{Z Z} E_{R R}+2 b_{6} E_{R R} E_{\Theta \Theta}$. 

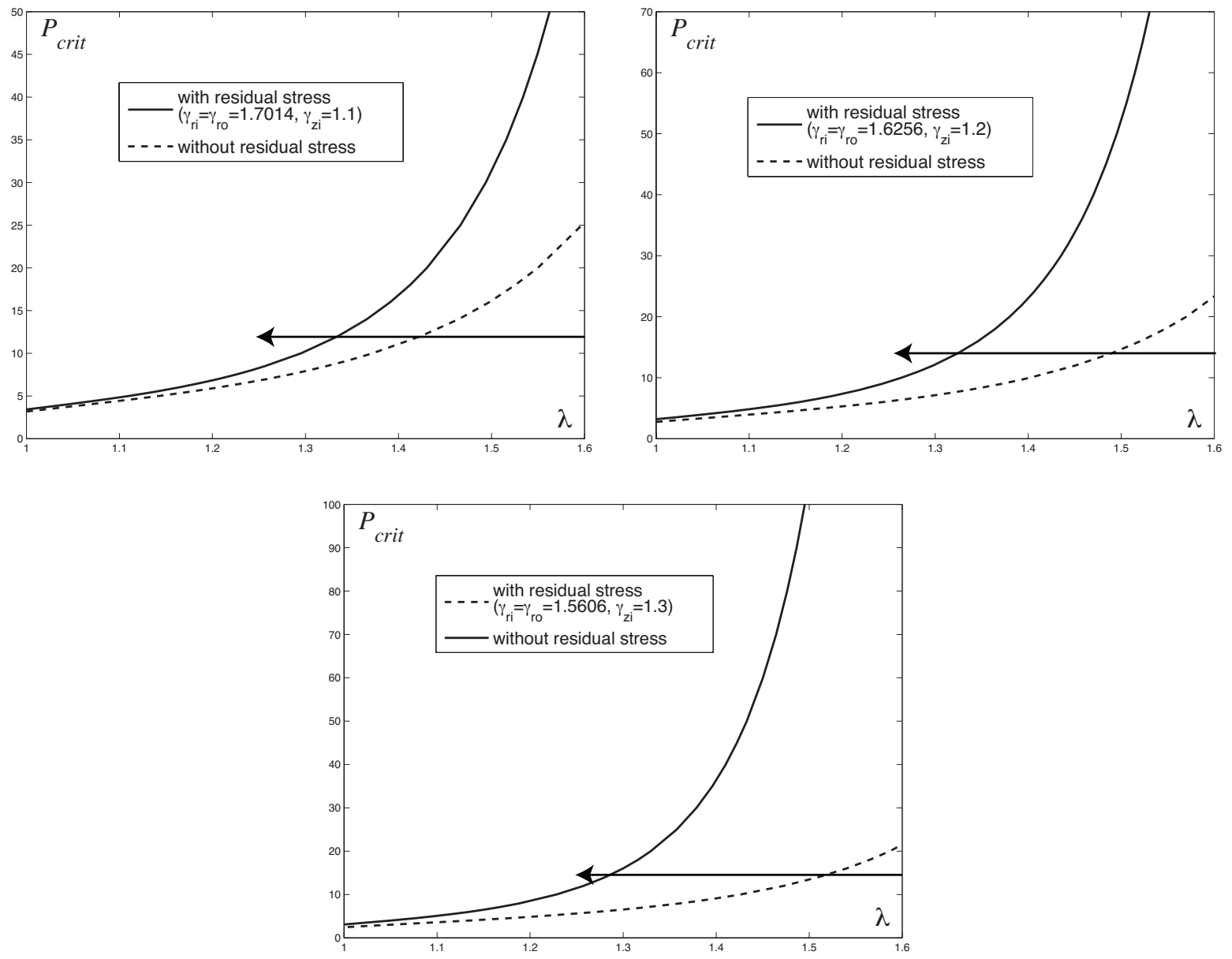

Figure 9: The critical internal pressure versus the axial stretch for various values of the differential axial growth. The arrow represents the release of axial loading resulting in a axial stretch of about 1.25 for $\mathrm{P}=13.3 \mathrm{kPa}$. In all cases, the artery becomes unstable. 



\section{RECENT REPORTS}

34/09 Tangent unit-vector fields: nonabelian homotopy invariants and the Dirichlet energy

Majumdar

Robbins

Zyskin

35/09 A metabolite-sensitive, thermodynamically-constrained model of cardiac cross-bridge cycling: Implications for force development during ischemia

Tran

Smith

Loiselle

Crampin

36/09 Modelling bacterial behaviour close to a no-slip plane boundary: the influence of bacterial geometry

Shum

Gaffney

Smith

Goswami

Pani imation to a second order linear parabolic initial and boundary value problem with nonsmooth initial data

38/09 Optimal L2 estimates for semidiscrete Galerkin methods for parabolic integro-differential equations with nonsmooth data

39/09 Spatially structured oscillations in a two-dimensional excitatory neuronal network with synaptic depression

40/09 Stationary bumps in a piecewise smooth neural field model with synaptic depression

41/09 Homogenization for advection-diffusion in a perforated domain

42/09 Fast stochastic simulation of biochemical reaction systems by alternative formulations of the Chemical Langevin Equation

43/09 Pseudoreplication invalidates the results of many neuroscientific studies

44/09 Cardiac cell modelling: Observations from the heart of the cardiac physiome project

45/09 A Hybrid Radial Basis Function - Pseudospectral Method for Thermal Convection in a 3-D Spherical Shell

46/09 Refining self-propelled particle models for collective behaviour

47/09 Stochastic Partial Differential Equations as priors in ensemble methods for solving inverse problems
Goswami

Pani

Yadav

Kilpatrick

Bressloff

Kilpatrick

Bressloff

Haynes

Hoang

Norris

Zygalakis

Melykuti

Burrage

Zygalakis

Lazic

Finka et al.

Wright

Flyer

Yates

Baker

Erban

Maini

Potsepaev

Farmer

Aziz 
48/09 DifFUZZY: A fuzzy spectral clustering algorithm for complex data sets

01/10 Fluctuations and instability in sedimentation

02/10 Determining the equation of state of highly plasticised metals from boundary velocimetry

03/10 Stability of bumps in piecewise smooth neural elds with nonlinear adaptation

04/10 Random intermittent search and the tug-of--war model of motordriven transport

05/10 Ergodic directional switching in mobile insect groups

06/10 Derivation of a dual porosity model for the uptake of nutrients by root hairs

07/10 Frost heave in compressible soils

08/10 A volume-preserving sharpening approach for the propagation of sharp phase boundaries in multiphase lattice Boltzmann simulations

09/10 Anticavitation and differential growth in elastic shells
Cominetti et al.

Guazzelli

Hinch

Hinch

Kilpatrick

Bressloff

Newby

Bressloff

Escudero et al.

Zygalakis

Roose

Majumdar

Peppin

Style

Sander

Reis

Dellar

Moulton

Goriely

Copies of these, and any other OCCAM reports can be obtained from:

Oxford Centre for Collaborative Applied Mathematics Mathematical Institute

24 - 29 St Giles'

Oxford

OX1 3LB

England

www.maths.ox.ac.uk/occam 